\title{
In Situ and Electron Tomography Observations of Dislocation-Defect Interactions in Ion Irradiated Austenitic Stainless Steels
}

\author{
J. Kacher,* G.S. Liu,* and I.M. Robertson* \\ *University of Illinois at Urbana-Champaign, Materials Science and Engineering Department, \\ Urbana, IL, 61801
}

Irradiation-induced mechanical property degradation appears as a loss of ductility and an increase in tensile and yield strength, which with increasing dose can lead to the emergence of a distinct yield drop even in face-centered cubic materials [1]. The presence of defect-free channels in deformed irradiated materials has been linked to this degradation and to irradiationassisted stress corrosion cracking. However, the evolution of these channels and how they influence macroscopic properties is still being debated. Previous in situ transmission electron microscopy (TEM) straining experiments by Briceno et al. on ion-irradiated 304 stainless steel illustrated a change in dislocation mobility due to irradiation defects that together with dynamic observation of defect-free channel formation suggested that dislocation accumulation at the exit side of the grain boundary could generate the local conditions for grain boundary cracking [2].

In the present work, two additional stainless steel alloys were studied with the main difference between them being their stacking-fault energy; the compositions and stacking-fault energies are given in Table 1. In the in situ experiments, two mechanisms for channel widening were observed: double cross-slip to avoid dislocation defect interactions and the nucleation of parallel dislocation sources at grain boundaries. An example of double cross-slip is shown in the micrograph presented in Figure 1. Note, however, double cross-slip was not reported to occur in the 304 stainless steel studied by Briceno et al. which may be due to ambiguities in interpretation arising from the two-dimensionality of electron micrographs. In order to clarify the spatial configuration associated with the double cross-slip shown in Figure 1, electron tomography was employed for post mortem analysis of the samples previously strained in situ; the results of this analysis will be presented and discussed. Other notable observations from the in situ experiments include: a segmented, jerky motion of the dislocations as they passed through the defect field; the production and passage of Shockley partial dislocations being restricted to defect-free channels first created by perfect dislocations; and the evolution of reverse dislocation pileups in grain interiors. The reverse dislocation pileup formation is attributed to the shear stress on the slip plane being insufficient to enable the leading dislocations to penetrate the obstacle field coupled with rapid generation of dislocations from the source.

The effects of this altered dislocation mobility and the resultant evolution of the microstructure will be discussed in terms of the local shear stresses on grain boundaries and slip systems. Implications of these results on the degradation of mechanical properties will be presented [4].

\section{References}

[1] Kirk, M. et al. Structural Mechanics in Reactor Technology. 2003: Prague, Czech Republic.

[2] Briceno, M., et al. Journal of Nuclear Materials. 2011. 409: p. 18-26

[3] Was, G., et al. Unpublished data. 2008. 
[4] This work was supported by the US Department of Energy, Offices of Basic Energy Sciences under contract DE-FG02-07ER46443 (electron tomography) and DE-FG02-08ER46525 (effects of ion irradiation).

Table 1. Composition of stainless steel alloys studied and their stacking fault energy (SFE) [3].

\begin{tabular}{|c|c|c|c|c|c|c|c|}
\hline Alloy & $\begin{array}{c}\mathrm{Cr} \\
\%\end{array}$ & $\begin{array}{c}\mathrm{Ni} \\
\%\end{array}$ & $\begin{array}{c}\mathrm{C} \\
\%\end{array}$ & $\begin{array}{c}\mathrm{Mn} \\
\%\end{array}$ & $\begin{array}{c}\mathrm{Si} \\
\%\end{array}$ & $\begin{array}{c}\mathrm{P} \\
\%\end{array}$ & $\begin{array}{c}\text { Meas. } \\
\text { SFE }\left(\mathrm{mJ} / \mathrm{m}^{2}\right)\end{array}$ \\
\hline 304 SS & 18.3 & 8.5 & 0.04 & 1.38 & 0.65 & 0.03 & 15.5 \\
\hline Alloy A & 13.41 & 15.04 & 0.016 & 1.03 & 0.1 & $<0.01$ & 36.3 \\
\hline Alloy B & 20.73 & 31.16 & 0.014 & 0.94 & 0.1 & 0.014 & 61.1 \\
\hline
\end{tabular}

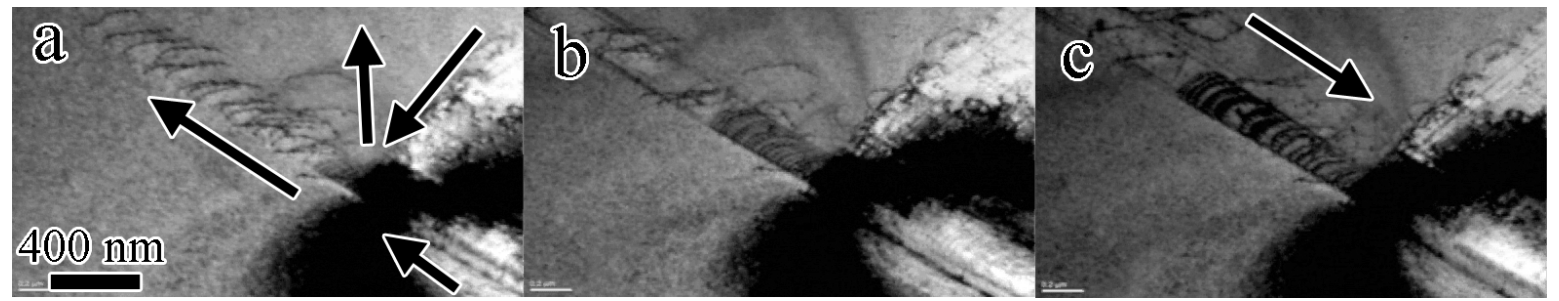

Figure 1. Reverse pileup formation from intersection of two slip systems in Alloy A. Time elapsed for each image is a) $0.0 \mathrm{~s}$, b) $6.0 \mathrm{~s}$, and c) $14.1 \mathrm{~s}$. Arrows denote direction of dislocation motion Notice that perfect dislocations progress in front and that pileup initially is packed densely at the emergence point from the intersection.

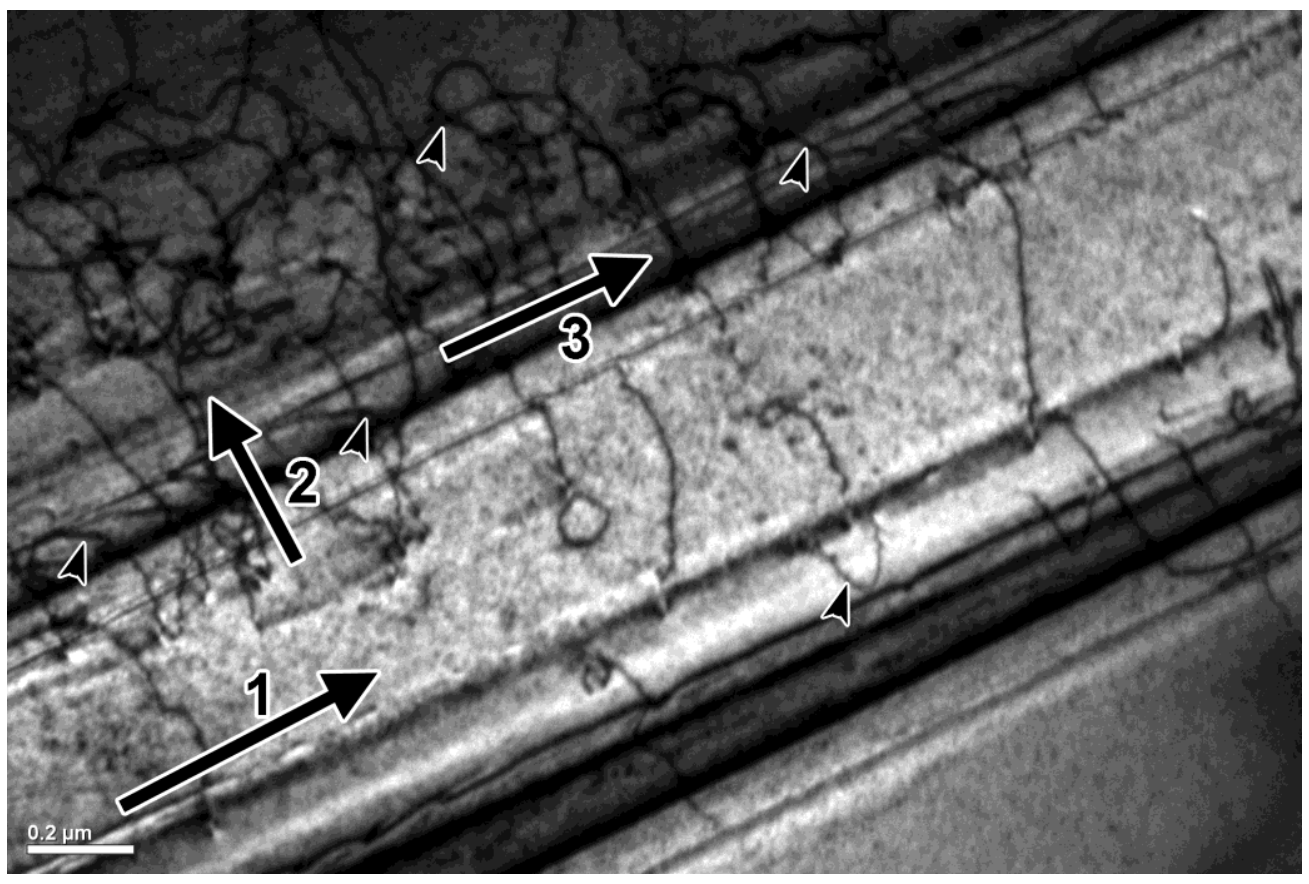

Figure 2. Dislocation cross-slip from defect-free channel in Alloy A. Higher intensity channel has been swept free of the majority of defects. The arrows denote 1) original dislocation motion through channel, 2) dislocation cross-slip from defect free channel, and 3) dislocation cross-slip back on to plane parallel to 1). Markers denote instances of dislocation cross-slip. 